vides an elegant, albeit cumbersome, method for testing this prediction. A more rapid, if less complete, approach would be to carry out the test in vitro; the ability to detect foreign peptide-independent, selfMHC recognition by antigen-specific $T$ cell clones and hybrids may provide the means to do so.

Although these new results and the model I have proposed address the molecular relationship between self- and foreign-peptide recognition by $\mathrm{T}$ cells, they do not resolve the central paradox of

\footnotetext{
1. Nikolić-Žugić, J. \& Bevan, M.J. Nature 344, 65-67 (1990).

2. Garrett, T.P.J., Saper, M.A., Bjorkman, P.J., Strominger J.L. \& Wiley, D.C. Nature 342, 692-696 (1989).

3. Schwartz, R.H. Cel/57, 1073-1081 (1989).

4. Claverie, J.-M. \& Kourilsky, P. Ann. Inst. Pasteur/Immun. 137D, 425-442 (1986).

5. Moore, M.W. Carbone, F.R. \& Bevan. M.J. Cell $\mathbf{5 4}$, 777-785 (1988).

6. Nathanson, S.G., et al. A. Rev. Immun. 4, 471 (1986)

7. Bjorkman, P.J. et al. Nature 329, 512-518 (1987)

8. Ogata, M. et al. J. Immun. 136, 1178-1185 (1986)

9. Singer, A. et al. Prog. Immun. VI, 60-66 (1986).

10. Sette, A. et al. Proc. natn. Acad. Sci. U.S.A. 86, 32963300 (1989).
}

thymic selection - the generation of a repertoire that is usable despite positive and negative selection events involving the same TCRs and MHC molecules. Many opinions have been expressed on this matter, but it remains the task of future experiments to unravel the paradox.

Ronald N. Germain is at the Laboratory of Immunology, National Institute of Allergy and Infectious Diseases. National Institutes of Health, Bethesda, Maryland 20892, USA.

11 Brown, J.H. et al. Nature 332, 845-850 (1988)

12. Davis, M.M. \& Bjorkman, PJ. Nature 334, 395-401 (1988)

13. Novotny, J., Bruccoleri, R.E. \& Kourilsky, P. Res. Immun 140, 145-158 (1989)

14. Ronchese, F., Brown, M.A. \& Germain, R.N. J. Immun 139, 629-638 (1987)

15. Ajitkumar, P et al Cell 54, 47-56 (1988)

16. Santos-Aguado, J., Crummins, M.A., Mentzer, S.J. Burakoff, S.J., \& Stromlinger, J.L. Proc. natn. Acad. Sci. U.S.A. 86, 8936-8940 (1989).

17. Schneck, J. etal. Proc. natn. Acad. Sci. U.S.A. 86,8516 8520 (1989).

18. Guillet, J.-G.,Lai, M.Z., Briner, T J., Smith, J.A. \& Gefter M.L. Nature 324, 260-262 (1986)

\title{
Switching off an after-effect
}

\section{Oliver Braddick}

Cognitive psychologists' accounts of visual information processing usually distinguish between 'pre-attentive' processes, which proceed independently of the subject's active control, and 'postattentive' processes, whose operation is determined by what component of the visual input has been actively selected. Increasingly neurophysiologists have been looking for modulations of neural activity in awake animals which might reflect this selective control. Recent psychophysical experiments reported by Chaudhuri on page 60 of this issue ${ }^{1}$ imply that the primary processing of visual motion information, which in most schemes would be placed firmly on the pre-attentive side of this divide, is in fact under the control of selective attention.

In the motion after-effect (MAE), prolonged vicwing of a moving region induces a strong sensation of opposite motion in a stationary test pattern which is subsequently viewed. This is believed to reflect adaptation of the responses in directionally selective neurons, as has been demonstrated in the visual pathway of rabbits ${ }^{2}$ and cats $^{3}$. Chaudhuri shows that if subjects have to monitor a sequence of rapidly changing letters and numbers, the MAE produced by a surrounding field of moving spots is reduced in duration by almost 70 per cent, compared to tests when the character sequence is still present and fixated but requires no action.

The most obvious, and probably the most important, mechanism of visual selective attention is the control of foveal fixation. But so much interest centres on the possibility of selective processes acting directly within the visual pathway, that the control of visual input by eye movements is generally regarded in this field as a disturbing experimental artefact. Chaudhuri recorded the subject's eye movements and showed that the attentional task did not change them enough to account for his results. More positively, attention could modify the MAE even when the subjects were required to attend to a location different from the position they were fixating.

The selective process demonstrated in these experiments is spatial; attention is devoted to one region of the visual field at the expense of another. It is also possible to select one attribute of a visual stimulus over another at the same location - for example to respond to colour rather than to shape or movement. Chaudhuri tested for such an effect by requiring his subjects to monitor the rapidly changing colour of the moving random-dot display, but this had no effect on the MAE. It is plausible that the neural basis of selection may be different for selecting a location and for selecting an attribute; the fact that visual areas are interconnected, topographical maps of the visual field is suggestive for possible mechanisms in the case of location. Clear analogues of spatial selection have been seen in the responses of single neurons ${ }^{4.5}$; although modulations of the relative selectivity of single neurons for colour versus pattern have been reported ${ }^{6}$, they are not always clear cut? ${ }^{7}$. But it is likely that the colour and motion properties of the stimulus are processed by distinct cortical streams ${ }^{8}$, so selection between them may well reflect different processes from those operating within the representation of the visual field in a single cortical area.

The studies of attention modulating neural responses have concentrated on extrastriate cortical areas (V4, IT) in the temporal stream of processing. Little effect of attention has been demonstrated in the primary striate cortex ${ }^{9}$, and Chaudhuri uses this to suggest a post-striate contribution to the MAE. Primary directional selectivity arises both in striate cortex and (at least for higher velocities) in the post-striate area MT (ref. 10); so his idea is not unreasonable, although it is perhaps surprising that the post-striate contribution should be so large at the relatively low velocities tested. But the processing stream leading to MT includes its own specific population of striate cells and is separate from the temporal stream in which attentional effects have been studied, so the anatomy of attention in relation to motion is still almost completely speculative.

Whatever the anatomy, Chaudhuri's results show that selective attention can modulate a process which must be regarded as coming early in the sequence of processing visual information. Early effects of selection, though, should not be confused with the long-standing psychological issue of an early or late site for the processes that determine selection. Anatomically, it is clear that the flow of information in the visual pathway cannot be one-way ${ }^{11}$, and a wide range of cortical structures may be able to modulate visual signals as early as the lateral geniculate body ${ }^{12}$. This 'top-down' control means that there may be little truly 'preattentive' processing beyond the retina. But that does not excuse us from the need to understand essential distinctions between the ways different visual processes are subject to, and require, active control.

Oliver Braddick is in the Department of Experimental Psychology, University of Cambridge, Cambridge CB2 $3 E B$, UK

1. Chaudhuri, A. Nature 344, 60-62 (1990)

2. Barlow, H.B. \& Hill, R.M. Nature 200, 1347 (1963).

3. Hammond, P., Mouat, G.S.V. \& Smith, A.T. Exp. Brain Res. 72, 1-20 (1988)

4. Moran, J. \& Desimone, R. Science 229, 782-784 (1985)

5. Sato, T. J. Neurophysiol. 60, 344-364 (1988)

6. Braitman, D.J. Brain Res, 307, 17-28 (1984)

7. Maunsell, J.H.R., Schiller, P.H. \& Hochstein, S. Pap. presented at Symp. Comp. Mod. Hum. Vis. (Center for Visual Science, University of Rochester, 1986)

8. De Yoe, E.A. \& Van Essen, D. Trends Neurosci. 11, 219-226 (1987).

9. Hänny, P.E. \& Schiller, P.E. Exp. Brain Res. 69, 225244 (1988).

10. Mikami, A., Newsome, W.T. \& Wurtz, R.H. I Neurophy siol. 55, 1308-1327 (1987).

. Zeki, S. \& Shipp, S. Nature 335, 311-317 (1988)

12. Crick, F.H.C. Proc. natn. Acad. Sci. U.S.A. 81, 45864590 (1984). 